\title{
FISIOTERAPIA - ORAL - 20/09/05
}

\section{4 \\ Benefício da resistência muscular localizada como otimização da atividade física programada. \\ Barbosa, Luciana de Castro, Bündchen, D.C., Richter, C.M., Pereira, A.R., Dipp, T., Panigas, T., Rubin, A.C., Barbosa, E.G., Viecili, P.R.N. \\ Instituto de Cardiologia de Cruz Alta - Centro Terapêutico Cruz Alta RS BRASIL.}

INTRODUÇÃO: Participação em programas de exercício físico (PEF) tem sido uma alternativa para atenuar os efeitos do sedentarismo. O PEF seria ideal se fosse composta tanto por exercícios aeróbicos, quanto por exercícios musculares, nos quais se enquadram os de resistência muscular localizada (RML) que são importantes para o aumento da massa muscular magra, do tônus e do equilíbrio muscular. OBJETIVO: Verificar a eficácia de um programa de exercícios de RML (PERML) no aumento da força muscular (FM) de indivíduos sedentários como complemento de um PEF.

METODOLOGIA: Empregou-se uma PEF em 22 indivíduos sedentários, sendo 9 homens ( $56 \pm 8$ anos) e 13 mulheres ( $58 \pm 6$ anos). O PEF foi realizado $3 x /$ semana, por 3 meses, com duração de até 50' sendo composto por 3 etapas: alongamento, exercício aeróbico e exercícios de RML. A intensidade de treinamento da atividade aeróbica foi de 60 a $75 \%$ do VO2 me consumo máximo de oxigênio indireto. Para os exercícios de RML, utilizou-se uma máxima (1RM). Foram mensurados os grupos musculares, bíceps, peitoral, dorsal, quadríceps e isquiotibiais. Os dados foram analisados através da $M \pm D P, D \%$, teste $t$ de Student, considerando $p<0,05$ significativo.

RESULTADOS: Houve aumento significativo no V02 máx. tanto dos homen $(27,54 \times 36,21 \mathrm{ml} / \mathrm{Kg} / \mathrm{min} ; \mathrm{p}<0,006)$ quanto das mulheres $(20,66 \times 27,16 \mathrm{ml} / \mathrm{Kg}$ min; $p=0,001)$. Um aumento da forca em todos os músculos, nas mulheres,

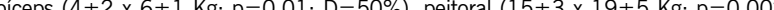
$D=27 \%)$, dorsat $(20 \pm 3 \times 25 \pm 6 \mathrm{Kg} ; \mathrm{p}=0,003 ; \mathrm{D}=25 \%)$, quadriceps $(15 \pm 4$ nos homens, bíceps $(8 \pm 2 \times 11 \pm 2 \mathrm{Kg} ; \mathrm{p}=0,002 ; \mathrm{D}=37,5 \%)$, peitoral $(25 \pm 4 \times$ $34 \pm 6 \mathrm{Kg} ; \mathrm{p}=0,003 ; \mathrm{D}=36 \%)$, dorsal ( $31 \pm 7 \times 38 \pm 6 \mathrm{Kg} ; \mathrm{p}=0,009 ; \mathrm{D}=22 \%$ ) quadríceps $(20 \pm 8 \times 34 \pm 5 \mathrm{Kg} ; \mathrm{p}=0,01 ; \mathrm{D}=70 \%)$, isquiotibiais $(21 \pm 4 \times 33 \pm 6 \mathrm{Kg}$;

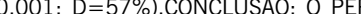
um aumento da FM tanto em homens quanto em mulheres, otimizando o PEF. carga de $40 \%$ da carga máxima, estipulada pelo teste de uma contração voluntária $19 \pm 4 \mathrm{Kg} ; \mathrm{p}=0,01 ; D=27 \%)$, isquiotibiais $(14 \pm 4 \times 18 \pm 3 \mathrm{Kg} ; \mathrm{p}=0,01 ; \mathrm{D}=28 \%)$ e

\section{5}

Alterações fisiológicas durante programa de reabilitação cardiovascular - fase I em pacientes submetidos à cirurgia cardíaca. Fernando de Souza Botega

UNIFESP-EPM SAO PAULO SP BRASIL.

INTRODUÇÃO: A fase hospitalar de reabilitação cardíaca tem como objetivo principal impedir ou minimizar a instalação de diversos efeitos deletérios do imobilismo além de tornar o pacientes hospitalizados auto-suficientes para a realização das atividades de vida diária no período mais breve possível.

OBJETIVO: Comparar o comportamento do aparelho cardiorespiratório antes, durante e após a realização do exercício físico em pacientes no pós-operatório (PO) em relação ao pré-operatório.

MÉTODO: Foi aplicado o protocolo de reabilitação cardíaca segundo a American Association of Cardiovascular \& Pulmonary Reabilitation (AACPR), em 14 pacientes submetidos à Cirurgia Cardíaca no pré-operatório, 3o e $40 \mathrm{PO}$, sendo estes dados analisados entre si.

RESULTADOS: Foram observadas, antes e após a realização de exercícios físicos, no 3o PO em comparação com o período pré-operatório, elevação da freqüência cardíaca $(p<0,001)$ e freqüência respiratória $(p<0,02)$, queda da pressão sistólica $(p<0,145)$, pressão diastólica $(p<0,116)$ e saturação de oxigênio $(p<0,001)$ e manteve-se ainda as notas dadas a escala de percepção de esforço BORG, após o protocolo $(p<0,09)$. No 40 PO os parâmetros analisados apresentaram discretas variações em seus resultados, sem significância estatística, sugerindo a melhora de desempenho físico.

CONCLUSÃO: A Cirurgia Cardíaca produz, de forma inicial, perda aguda do condicionamento físico. No entanto os pacientes introduzidos precocemente (Fase I) ao programa fisioterapêutico de reabilitação cardíaca parecem apresentar pequena melhora de seu desempenho físico, apesar de não atingirem os valores referenciais do período pré-operatório, sendo isto esperado nas fases II e III.

O teste da caminhada de 6 minutos pós-infarto agudo do miocárdio. Nogueira PAMS, Leal AC, Pulz C, Nogueira IDB, Salles AF, Salvetti XM, Oliveira Fo JA. UNIFESP - EPM São PaUlo SP BRASIL.

FUNDAMENTO: 0 teste da caminhada de 6 minutos (TC6 min) é um teste submáximo, amplamente utilizado na doença pulmonar e em miocardiopatias para avaliação da tolerância ao esforço físico (American Association of Cardiovascular \& Pulmonary Rehabilitation-AACPR, 1999). No entanto, não há relatos sobre a utilização do TC6 min pós-infarto agudo do miocárdio (IAM). OBJETIVO: Investigar a reprodutibilidade, a exeqüibilidade e os riscos do TC6 min pós-IAM, sob monitorização eletrocardiográfica contínua.

DEFINIÇÃO: Estudo transversal.

PACIENTE: Avaliaram-se 25 pacientes em uso de medicação, dos quais 23 homens, $54 \pm 8$ anos, $5 \pm 0,9$ dias pós-IAM. Em 20 pacientes, 18 homens (44-72 anos) com teste ergométrico prévio normal, avaliou-se a reprodutibilidade do TC6 min. MÉTODOS: O protocolo foi aprovado pela Comissão de Ética da Instituição. Foi aplicado o TC6 min (protocolo da AACPR) em 3 ocasiões, com intervalo de 1 hora, tendo as variáveis cardiorespiratórias alcançado os valores de repouso. Avaliaram-se ECG (Sistema Holter), freqüência cardíaca (FC- bpm), pressão arterial (PA- $\mathrm{mmHg}$ ), freqüência respiratória (f- $\mathrm{rpm}$ ), duplo produto (DP bpm.mmHg), percepção de esforço (PE - Borg 6 a 20) e distância percorrida no TC6 min (d- m). Calculou-se o coeficiente de correlação intraclasses ( $r$ ).

RESULTADOS: Em todos os casos os testes foram completados sem complicações. Não se detectaram sintomas e sinais significativos, arritmias complexas e alterações significantes em segmento ST. Verificou-se reprodutibilidade ao final dos testes nas medidas de PA sistólica $(r=0,93)$, PA diastólica $(r=0,95), f(r=0,88)$, DP $(r=0,91)$, PE $(r=0,94)$ e d $(r=0,82)$. Registraram-se arritmias grau I (escala de Lown), em respectivamente, $15 \%, 15 \%$ e $5 \%$ dos TC6 min, que, em geral, não se repetiram no mesmo paciente.

CONCLUSÕES: Os autores ressaltam a segurança, a reprodutibilidade e exeqüibilidade do TC6 min no pós-IAM em pacientes com TE prévio normal.

\section{7 \\ Alterações hemodinâmicas causadas pela manuvacuometria observadas ao ecocardiograma em pacientes no pré-operatório} de cirurgia de revascularização do miocárdio.

Raquel Franchin Ferraz, Kelly Cristiani Tavolaro, Gerson Cipriano Junior, Aurelio carvalho pinheiro, Roberto Catani, Enio Buffolo.

Universidade Federal de São Paulo São Paulo SP BRASIL.

INTRODUÇÃO: A manuvacuometria é utilizada para verificar eficácia ventilatória em cardiopatas no pré-operatório cirurgia de revascularização do miocárdio (RM). Estudos descrevem haver transmissão dessas pressões à cavidade pericárdica, o que pode alterar o relaxamento do ventrículo esquerdo (VE). A ecocardiografia é um método confiável, prático e não-invasivo para diagnóstico e acompanhamento de pacientes com disfunção de VE. Este exame avalia padrão de fluxo mitral que se correlaciona com a performance diastólica do VE. OBJETIVO: Verificar a presença de alterações hemodinâmicas durante a manuvacuometria em pacientes no pré-operatório de cirurgia de RM.

MÉTODO: Foi realizada manuvacuometria máxima com manobras sustentadas por 5 segundos e, simultaneamente, foi realizada a avaliação de VE através do ecocardiograma.

RESULTADOS: Foram avaliados 7 pacientes ( 6 homens; 1 mulher) com idade entre 52 e 73 anos. Todos os indivíduos apresentavam um padrão de menor relaxamento de VE e diminuição na fase de enchimento ventricular rápido ao repouso, mantido durante a PImax e exacerbado durante a PEmax, a fração de ejeção apresentou queda na fase inspiratória (45\% para $42 \%$ ) e aumento na fase expiratória (45\% para $49 \%$ ). As pressões encontradas foram PImax = $81 \mathrm{mmHg}(\mathrm{DP}=28,84)$ e PE máx $=76 \mathrm{mmHg}(\mathrm{DP}=20,99)$.

CONCLUSÃO: Observamos que os pacientes portadores de doença arterial coronariana possuem um padrão de enchimento ventricular esquerdo com alteração de relaxamento, o qual é mantido ou exacerbado durante a avaliação de manuvacuometria. Tal fato pode sugerir menor perfusão do miocárdio durante a avaliação. 


\section{8}

Influência dos turnos matutino vespertino no comportamento hemodinâmico cardiovascular em programa de exercício físico.

Pereira, Angela Maria, Richter, C., Barbosa, L., Bündchen, D., Panigas, T., Dipp, T., Rubin, A. Barbosa, E., Viecili, P. R.

Instituto de Cardiologia de Cruz Alta - Centro Terapêutico Cruz Alta RS BRASIL.

OBJETIVO: Verificar se há diferenças no comportamento hemodinâmico durante Exercício Físico (EF) realizada nos turno matutino vespertino.

MÉTODOS: 24 indivíduos sedentários divididos em dois grupos iguais, 7 mulheres e 5 homens. 0 grupo matutino (GM: $61,3 \pm 4,7$ anos) realizou EF às 9 horas $e$ o grupo vespertino (GV: $54 \pm 8$ anos) às 17 horas. Foram avaliados através do teste ergométrico com protocolo de Bruce para obtenção indireta do consumo de oxigênio (VO2máx.), com 50 a 75\%. O EF constou de 3 etapas: alongamento, atividade aeróbica e muscular, $3 \times$ /semana, por 3 meses. Mensurou-se pressão arterial sistólica e diastólica inicial (PASI e PADI), aeróbica (PASA e PADA) e final (PASF e PADF), freqüência cardíaca inicial (FCI), aeróbica (FCA) e final (FCF) e V02máx. Analisou-se os dados por $M \pm D P$, teste $t$, sendo significativo $p<0,05$. RESULTADOS: A PASI no GV apresentou valores pressóricos superiores ao GM $(123,5 \pm 18,5 \times 121,4 \pm 16 \mathrm{mmHg} ; \mathrm{p}<0,03)$, a PASA foi superior no $\mathrm{GM}$ ao GV $(137,8 \pm 18,3 \times 129,7 \pm 21 \mathrm{mmHg} ; p<0,001)$, na PASF os dois grupos não apresentaram diferenças $(121 \pm 14 \times 121,5 \pm 19)$. A PADI no GV apresentou valores superiores ao GM $(77,8+11 \times 75,6+9 \mathrm{mmHg} \cdot \mathrm{p}<0,008)$, a PADA permaneceu igual nos grupos $(78,3 \pm 10 \times 78,6 \pm 10 \mathrm{mmHg})$. Na PADF o GV apresentou valores superiores ao $\mathrm{GM}(77,5 \pm 10 \times 75,8 \pm 8,5 \mathrm{mmHg} ; \mathrm{p}<0,002)$. Na FCl o GM apresentou valores superiores ao GV $(80,7 \pm 11 \times 78 \pm 12$ bpm;p<0,005). A FCA e FCF permaneceram iguais nos grupos $(104,9 \pm 12 \times 104,5 \pm 13 \mathrm{bpm}$ $84,2 \pm 12 \times 84,7 \pm 12 \mathrm{bpm}$, respectivamente). O VO2 tanto no GM quanto no GV apresentaram valores inciais e finais similares $(23 \pm 9 \times 25 \pm 8 \mathrm{ml} . \mathrm{kg} / \mathrm{min} ; 30,7 \pm$ $8 \times 34,2 \pm 7,6 \mathrm{ml} . \mathrm{kg} / \mathrm{min}$.). Ambos obtiveram aumento significativo do VO2 máx. após o PAF (GM:23 $\pm 9 \times 30,7 \pm 8 \mathrm{ml} . \mathrm{kg} / \mathrm{min}$.;GV:25 $\pm 8 \times 34 \pm 7,6 \mathrm{ml} . \mathrm{kg} / \mathrm{min})$.

CONCLUSÃO: Apesar do GV iniciar os exercícos com níveis pressóricos mais elevados que o GM, os valores sistólicos finais foram semelhantes. Já os níveis diastólicos pré e pós atividade do GV se mostraram semelhantes, no entanto superiores no GM, porém não houve diferença no comportamento hemodinâmico em diferentes turnos.

\section{0}

A importância do treinamento prévio no uso do desfibrilador externo automático por fisioterapêutas e enfermeiros.

Carlos Cezar I. S. Ovalle.

Unidade de Terapia Intensiva - HC - UNICAMP Campinas SP BRASIL.

INTRODUÇÃO: A pronta instituição das manobras de ressuscitação cardiopulmonar (RCP) e o uso do desfibrilador externo automático (DEA) por profissionais não médicos tem se mostrado altamente eficaz no atendimento de vítimas de parada cardiorespiratória (PCR) intra-hospitalar. Porém, no Brasil, este procedimento ainda não foi analisado, o que justificou e estimulou a realização desta pesquisa.

OBJETIVO: Avaliar a importância do treinamento prévio na utilização do DEA por fisioterapeutas e enfermeiros em hospitais gerais.

MÉTODO: Fisioterapeutas e enfermeiros realizaram a simulação do atendimento de uma PCR utilizando o DEA. Grupo I: composto por profissionais com treinamento prévio para o uso do DEA e o Grupo II: sem o treinamento. O cenário foi montado utilizando o manequim AmbuR Man e o desfibrilador Medtronic Physio Control LifepakR 500 de treinamento. Os procedimentos foram avaliados de acordo com o protocolo de utilização do DEA da American Heart Association, incluindo o tempo de chegada no leito até a aplicação do primeiro choque, a colocação correta das pás adesivas e o tempo total de atendimento.

RESULTADOS: 0 grupo I apresentou melhor tempo resposta $(P<0,001)$ e melhor desempenho $(P<0,001)$ no atendimento cardíaco de emergência quando comparado ao grupo II.

CONCLUSÃO: No ambiente hospitalar, indivíduos previamente treinados apresentam melhores condições de realizar o atendimento cardíaco de emergência, incluindo o uso do DEA, que aqueles não treinados.

\section{9}

Influência de beta-bloqueadores na freqüência cardíaca de diabéticos hipertensos submetidos à atividade física.

Barbosa, Luciana de Castro., Pereira, A.M.R., Rubin, A.C., Richter, C.M., Bündchen, D.C., Barbosa, E.G., Dipp, T., Panigas, T., Viecili, P.R.N.

Instituto de Cardiologia de Cruz Alta - Centro Terapêutico Cruz Alta RS BRASIL.

INTRODUÇÃO: Portadores de Diabetes Mellitus podem evoluir com neuropatia autonômica resultando em freqüências cardíacas $(\mathrm{FC})$ mais elevadas. A hipertensão arterial sistêmica pode coexistir em diabéticos $(\mathrm{DH})$, onde muitos acabam usando beta-bloqueadores (BB) que tem efeito cronotrópico negativo. OBJETIVO: Verificar o comportamento da FC em indivíduos DH em uso de BB ao longo de um Programa de Exercícios Físicos (PEF). MÉTODOS: 12 indivíduos com 58 \pm 15 anos, sendo $50 \%$ masculino, divididos em 3 grupos iguais: $\mathrm{DH}$ em uso de $\mathrm{BB}$ $(80 \pm 37 \mathrm{mg} / \mathrm{d})$, não-diabético hipertensos (NDH) em uso de BB $(45 \pm 37 \mathrm{mg} / \mathrm{d})$ e sedentários hígidos (GC), submetidos à avaliação da capacidade funcional (VO2), através do teste ergométrico, pré e pós PEF. O PEF constou de treinamento de $70 \%$ da carga, 3x/semana, sessões aeróbicas até $40 \mathrm{~min}$. por 3 meses. Comparou-se a FC ao longo do PEF, considerando-se o repouso (FCR), pico máximo ( $F C M)$ e pós-atividade (FCP). Os dados foram analisados pela $M \pm D P$, teste $t$ de Student, ondep $<0,05$ foi singnificativo. RESULTADOS: Não houve um aumento significativo no VO2 no grupo DH $(24,1 \pm 6 \times 30,5 \pm 7 \mathrm{ml} / \mathrm{kg} / \mathrm{min} ; \mathrm{p}=0,1)$, porém observou-se um aumento no NDH $(16,3 \pm 5 \times 24,1 \pm 5 \mathrm{ml} / \mathrm{kg} / \mathrm{min} ; \mathrm{p}=0,01)$ e no GC $(23,6 \pm 9 \times 31,9 \pm 8 \mathrm{ml} / \mathrm{kg} / \mathrm{min} ; \mathrm{p}=0,004)$. A FCR do DH apresentou-se mais elevada quando comparada ao grupo $\mathrm{NDH}(70 \pm 14 \times 61 \pm 8 \mathrm{bpm} ; \mathrm{p}=0,003)$ e menor quando comparada ao GC $(70 \pm 15 \times 86 \pm 11$ bpm; $p=0,009)$. 0 grupo $\mathrm{NDH}$ apresentou menor FCR do que o GC $(61 \pm 8 \times 86 \pm 11 \mathrm{bpm} ; \mathrm{p}=0,002)$. Quanto a FCM o grupo $\mathrm{DH}$ apresentou uma FC mais elevada que o grupo $\mathrm{NDH}(90 \pm 17$ x 79 $\pm 12 \mathrm{bpm} ; \mathrm{p}=0,0008)$, e menor que o GC $(90 \pm 17 \times 109 \pm 12 \mathrm{bpm} ; \mathrm{p}=0,002)$. $\mathrm{NDH}$ apresentou menor FCM do que o GC $(79 \pm 12 \times 109 \pm 12 \mathrm{bpm} ; \mathrm{p}=0,003)$. 0 grupo $\mathrm{DH}$ apresentou uma $\mathrm{FCP}$ mais elevada que o grupo dos $\mathrm{NDH}(72 \pm 17 \times$ $67 \pm 9$ bpm; $p=0,001)$ e menor quando comparada ao GC $(72 \pm 17 \times 92 \pm 12$ $\mathrm{bpm} ; \mathrm{p}=0,006)$. NDH apresentou a FCP menor que o GC $(67 \pm 9 \times 92 \pm 12 \mathrm{bpm}$; $\mathrm{p}=0,002)$. CONCLUSÃO: O grupo $\mathrm{DH}$ apresentou $\mathrm{FC}$ maior no repouso, no pico do exercício e pós- atividade quando comparado com $\mathrm{NDH}$, apesar de maior dosagem de $\mathrm{BB}$ e menor do que o GC. Essa diferença provavelmente se deva à neuropatia autonômica.

\section{1}

Efeitos do Óxido Nítrico inalatório em pacientes com hipertensão pulmonar no pós-operatório de lesões valvares mitrais.

Ana Paula F. Becker, Renato A.K. Kalil, Edemar Pereira, Luciana Tosetto, André Dias Bueno, Carlos Antônio Vieira, Paulo Prates, Ivo A. Nesralla.

Instituto Cardiologia RS/Fundação Universitária Cardiologia Porto Alegre RS BRASIL.

A hipertensão pulmonar constitui um problema que pode levar a uma parte substancial da morbi-mortalidade no pós-operatório de cirurgia cardíaca. 0 objetivo deste trabalho consiste em verificar possíveis alterações na pressão arterial pulmonar (PAP), dentre outros fatores, durante a utilização do óxido nítrico inalatório (NOi) em pacientes no pós-operatório de lesões valvares mitrais. METODOLOGIA: Foram estudados 20 pacientes com diagnóstico prévio de hipertensão pulmonar secundária à lesão mitral (PAP sistólica pré-operatória maior ou igual a $60 \mathrm{mmHg}$ ). A dose de NOi utilizada foi de $20 \mathrm{ppm}$. Os pacientes eram monitorados periodicamente em relação à pressão arterial média, pressão venosa central, pressão média de átrio esquerdo, oximetria de pulso e complacência pulmonar estática. Foram realizadas medidas de PAP através de catéter de artéria pulmonar antes da utilização do NOi, 30 minutos após o início do tratamento e a cada 6 horas até a descontinuidade do tratamento, conforme critérios clínicos. RESULTADOS: o tempo mediano de utilização do NOi foi de 19,1 horas. A PAP média caiu significativamente de $33,8 \mathrm{mmHg}$ (pré-NOi) para $29,1 \mathrm{mmHg}$ nos 30 min iniciais e para $28,4 \mathrm{~mm}$ Hg considerando a média de todas as medidas pós NOi $(p<0,001)$. Não houve alterações significativas nas demais medidas hemodinâmicas.Conclusão: Os achados sugerem que a utilização do NOi reduz a PAP sem efeitos sistêmicos, demonstrando ser um recurso eficaz para o manejo da hipertensão pulmonar no pós-operatório de lesões valvares mitrais. Acreditase que sua utilização ainda não se tornou uma unanimidade devido, dentre outros fatores, à sua variabilidade de resposta. 


\section{2}

Descontinuação da ventilação mecânica no pós-operatório de cirurgia cardíaca:Peça T e PSV são realmente semelhantes?

Catia Coimbra, Juliana Paola, Lilian Kelly Oliveira, Juliani Goulart, Claudia Cadille, Rafael Braga, Rodrigo Rivelino ( acadêmico ), Valdenia Pereira de Souza, Monclar Policarpo. Hospital de Clínicas de Niterói Niterói RJ BRASIL.

OBJETIVOS: Avaliar a eficácia do emprego da Peça T e o PSV na descontinuação da ventilação mecânica em pacientes (pac), submetidos a cirurgia cardíaca não complicada.

MÉTODOS: 77 pac consecutivos submetidos a cirurgia cardíaca foram aleatoriamente divididos em dois grupos: gupo 1 (GI)- pac submetidos ao protocolo de descontinuação com Peça T e grupo 2 (GII)-pac submetidos ao protocolo com PSV. Radiografia de tórax foi realizada em todos os pacientes previamente a retirada do TOT. Pacientes foram extubados segundo critérios convenciais de nível de consciência, força muscular e parâmetros gasométricos. RESULTADOS: Circulação extracorpórea foi empregada em $89 \%$ dos pac do G e $94 \%$ dos pac do GII ( $P=N S$ ). A média de idade foi de 59 anos no Gl e 62 anos no GII $(P=N S)$. Não há diferença significativa com relação a sexo, resultados gasométricos e tempo de CEC entre os dois grupos. Houve registro de 27 atelectasias sendo 06 no GI e 21 no GII. O tempo de descontinuação da ventilação foi de $4: 35 \mathrm{~h}$ no $\mathrm{Gl}$ e $5: 40$ no Gll.

CONCLUSÃO: Ambas as técnicas se mostraram eficazes, mas o número de atelectasias e o tempo de permanência na prótese ventilatória foi maior no GII (PSV).

\section{3}

Avaliação de fatores de risco operatório que influenciam no desmame da ventilação mecânica de pacientes submetidos à cirurgia cardíaca e complicações respiratórias pós-operatórias.

Almeida, GF, Alcântara, TO, Guimarães, E, Menezes, JV, Nogueira, ACD.

Biocor Instituto Nova Lima MG BRASIL.

FUNDAMENTO: A extubação precoce no pós-operatório (PO) é um objetivo primordial na redução da morbi-mortalidade dos pacientes. 0 uso de variáveis clínicas pré, inter e pós-operatórias torna-se útil no prognóstico do tempo de ventilação mecânica (VM) e incidência de complicações pós cirurgia cardíaca.

OBJETIVO: Avaliar variáveis clínicas operatórias associadas à ventilação mecânica prolongada (VMP) e complicações no PO de cirurgia cardíaca eletiva.

DEFINIÇÃO: Estudo observacional, prospectivo, de análise univariada.

MATERIAL: Quinhentos pacientes adultos: 313 homens e 187 mulheres, divididos em 2 grupos. 0 grupo de VMP com 29 pacientes e o grupo sem VMP com 41 pacientes (selecionados aleatoriamente do total de 500).

MÉTODOS: Estudo realizado no Biocor Instituto, sendo estudadas as seguintes variáveis: idade, sexo, tabagismo, patologias prévias, complicações e procedimentos operatórios, além de óbitos e tempo de VM (prolongado quando $\geq 2$ dias).

RESULTADOS: 0 fator de risco prévio significativo em relação à VMP foi a insuficiência cardíaca congestiva (ICC) (Odds ratio 4,40). No grupo de VMP, 16 $(3,2 \%)$ pacientes evoluíram ao óbito. Dezessete pacientes $(3,4 \%)$ foram reintubados e $10(2 \%)$ traqueostomizados. A VMP esteve associada com óbito $(p<0,001)$, hipoxemia $(p=0,007)$ e insuficiência renal aguda no PO ( $p=$ 0,001 ). A associação de complicações respiratórias com VMP foi estatisticamente significativa $(p<0,001)$, sendo a pneumonia a mais freqüente.

CONCLUSÕES: A VMP está associada com ICC prévia e aumento de complicações pós-operatórias e óbitos nos pacientes submetidos à cirurgia cardíaca.

\section{4}

Avaliação da correlação entre a sexo e o tempo de ventilação mecânica no pós operatório de cirurgia cardíaca.

Juliana Alegre, Maira Bezerra Silva, Gisele Botelho Gonçalves, Leila Macias Kirk, Sergio Araujo Olival, Braulio Santos.

Instituto Nacional de Cardiologia Laranjeiras Rio de Janeiro RJ BRASIL.

FUNDAMENTOS: 0 tempo de assistência ventilatória é um marcador de morbidade em cirurgia cardíaca. Portanto, é essencial conhecer os fatores que estão associados à necessidade aumentada da ventilação mecânica nos diferentes sexos.

OBJETIVO: Avaliar, nesta população, os fatores que estão associados à necessidade de tempo de ventilação superior a $24 \mathrm{~h}$ entre os pacientes submetidos à cirurgia de revascularização (R) e/ou troca valvar (V) nos sexos feminino e masculino.

MATERIAL E MÉTODOS: Foram analisados 264 pacientes consecutivos submetidos à cirurgia de revascularização e/ou troca valvar no período entre $01 / 2004$ à $01 /$ 2005. As variáveis de exposição foram: sexo, idade ( $<70$ anos ou $>70$ anos), tipo de cirurgia $(R, V$ ou $R+V$ ) e complicações pós-operatórias (sangramento e vasoplegia). A variável de desfecho foi tempo de assistência ventilatória superior a 24h. Os testes estatísticos utilizados foram Qui-quadrado e o teste exato de Fisher. RESULTADOS: A população estudada tinha mediana de 60 anos com intervalo interquartil de 49 à 69 anos; 98 (37,12\%) eram mulheres e 160 foram R, $91 \mathrm{~V}$ e $13 \mathrm{R}+\mathrm{V}$. Não encontramos associação estatisticamente significativa entre tempo de ventilação superior a $24 \mathrm{~h}$ e as seguintes variáveis: sexo, idade, tipo de cirurgia e sangramento. Vinte e três $(44,23 \%)$ dos 52 pacientes que tiveram vasoplegia e $35(19,77 \%)$ dos 212 sem vasoplegia tiveram tempo de ventilação superior a $24 \mathrm{~h}(\mathrm{p}=0,00002)$.

CONCLUSÃO: Não há diferença estatisticamente significativa entre os sexos nos pacientes submetidos à cirurgia cardíaca no tempo de ventilação mecânica.

\section{5}

Avaliação das complicações que prolongam o tempo de ventilação mecânica no pós operatório de cirurgia cardíaca.

Juliana Alegre, Gisele Botelho Gonçalves, Leila Macias Kirk, Maira Bezerra Silva, Braulio Santos, Sergio Araujo Olival.

Instituto Nacional de Cardiologia Laranjeiras Rio de Janeiro RJ BRASIL.

FUNDAMENTOS: 0 tempo de assistência ventilatória é um marcador de morbidade em cirurgia cardíaca. Portanto, é essencial conhecer os fatores que estão associados a necessidade aumentada da ventilação mecânica.

OBJETIVO: Avaliar, nesta população, os fatores que estão associados à necessidade de tempo de ventilação superior a $24 \mathrm{~h}$ entre os pacientes submetidos a cirurgia de revascularização (R) e/ou troca valvar (V).

MATERIAL E MÉTODOS: Foram analisados 264 pacientes consecutivos submetidos à cirurgia de revascularização e/ou troca valvar no período entre 01/2004 à 01/2005. As variáveis de exposição foram: sexo, idade ( $<70$ anos ou $>70$ anos), tipo de cirurgia $(R, V$ ou $R+V)$ e complicações pós-operatórias (sangramento e vasoplegia). A variável de desfecho foi tempo de assistência ventilatória superior a 24h. Os testes estatísticos utilizados foram Qui-quadrado e o teste exato de Fisher.

RESULTADOS: A população estudada tinha mediana de 60 anos com intervalo interquartil de 49 à 69 anos; $98(37,12 \%)$ eram mulheres e 160 foram R, $91 \mathrm{~V}$ e 13 R+V. Não encontramos associação estatisticamente significativa entre tempo de ventilação superior a $24 \mathrm{~h}$ e as seguintes variáveis: sexo, idade, tipo de cirurgia e sangramento. Vinte e três $(44,23 \%)$ dos 52 pacientes que tiveram vasoplegia e $35(19,77 \%)$ dos 212 sem vasoplegia tiveram tempo de ventilação superior a $24 \mathrm{~h}(\mathrm{p}=0,00002)$.

CONCLUSÃO: A vasoplegia determina aumento do tempo de assistência ventilatória em pacientes submetidos à cirurgias cardíaca. 